\title{
Quantum optics laboratories for undergraduates
}

\section{Mark Beck, Ethan Dederick}

Mark Beck, Ethan Dederick, "Quantum optics laboratories for undergraduates," Proc. SPIE 9289, 12th Education and Training in Optics and Photonics Conference, 92891G (17 July 2014); doi: 10.1117/12.2070525

SPIE Event: 12th Education and Training in Optics and Photonics Conference, 2013, Porto, Portugal 


\title{
Quantum Optics Laboratories for Undergraduates \\ Mark Beck* and Ethan Dederick \\ Department of Physics, Whitman College, Walla Walla, WA, USA 99362
}

\begin{abstract}
We have developed a series of undergraduate teaching laboratories that explore some of the fundamentals of quantum mechanics. All of the experiments involve performing measurements on individual photons or entangled-photon pairs. The experiments include: "Proving" that light consists of photons, single-photon interference, and tests of local realism. We will describe the experiments, placing an emphasis on an experiment which measures the quantum-mechanical polarization state of a photon. This experiment explicitly demonstrates that measurements performed on one member of an entangled-photon pair effect the results of measurements of the other photon. We will also describe how we have integrated the experiments with our upper-level undergraduate quantum mechanics course.
\end{abstract}

Keywords: quantum optics, quantum mechanics, quantum state measurement, laboratories, photons, entanglement.

\section{INTRODUCTION}

Technology has advanced to the point where it is possible to perform experiments that examine truly fundamental aspects of quantum mechanics in an undergraduate teaching laboratory at a reasonable cost [1-10]. Over the past decade a number of different experiments, all involving individual photons or entangled-photon pairs, have been adapted for teaching laboratories [1]. These experiments include: "Proving" that light consists of photons [2], single-photon interference [3,4], tests of local realism [5-7], and the Hong-Ou-Mandel interferometer [8].

At a previous conference we described in detail experiments involving single-photon interference [4]; here we will describe experiments which measure the full quantum polarization state of an individual photon. Such a state measurement is particularly interesting when performed on a photon which is one member of an entangled-photon pair. For an entangled state different measurements performed on the idler photon project the signal photon into different states. This interesting phenomenon can be readily observed by measuring the state of the signal photon, conditioned upon the result of a measurement of the idler photon. For students these measurements vividly illustrate an important aspect of entanglement: Measurements performed on one member of an entangled-photon pair effect the results of measurements performed on the other.

However, before describing the quantum state measurements, we'll describe how the laboratory experiments complement our upper-level undergraduate quantum mechanics course.

\section{QUANTUM MECHANICS COURSE}

Our course begins with a presentation of the classical theory of polarization, followed by a description of the corresponding quantum theory. Analogies between the two theories minimize conceptual difficulties that students typically have when first presented with quantum mechanics. Furthermore, because the laboratories involve studying photons, using photon polarization as a prototypical quantum system allows the laboratory work to be closely integrated with the coursework.

Polarization represents a two-dimensional quantum system, so the introduction to quantum mechanics uses twodimensional state vectors and operators. This allows students to become comfortable with the mathematics of a relatively simple quantum system before moving on to more complicated systems. After describing polarization, the course goes

*beckmk@whitman.edu; http://www.whitman.edu/ beckmk/ 
on to describe spin systems, time evolution, continuous variable systems (harmonic oscillator, hydrogen atom, etc.), perturbation theory, and brief introductions to field theory and quantum information. Other topics covered include quantum measurement and entanglement.

\section{QUANTUM STATE MEASUREMENT--THEORY}

The state of a quantum mechanical system determines the probabilities of the outcomes of measurements that can be performed on that system. In this sense it contains all of the knowable information about the system; if you know the state, you know the probability of any measurement you could perform on that system. Thus, in some sense, a state measurement is the ultimate measurement one can make on a quantum system. In this section we'll describe the theory of quantum state measurement of the polarization of a single photon. This discussion is based on that in Ref. [11].

The polarization state of a photon can be expressed in terms of a density operator $\hat{\rho}$. This operator can be expressed as a density matrix using the basis of horizontally $|H\rangle$ and vertically polarized photons $|V\rangle$ as

$$
\hat{\rho}=\left(\begin{array}{cc}
\langle H|\hat{\rho}| H\rangle & \langle H|\hat{\rho}| V\rangle \\
\langle V|\hat{\rho}| H\rangle & \langle V|\hat{\rho}| V\rangle
\end{array}\right)=\left(\begin{array}{cc}
A & C^{*} \\
C & B
\end{array}\right),
$$

where $A$ and $B$ are real quantities, and $C$ is complex. The density operator is normalized such that its trace is 1 :

$$
\operatorname{Tr}(\hat{\rho})=A+B=1 .
$$

We can perform a measurement of the polarization in the horizontal-vertical basis (HV-basis) by passing the beam through a polarization analyzer that splits the beam into its horizontal and vertical components $\left(\mathrm{a} \mathrm{PA}_{\mathrm{HV}}\right)$ and then counting the photons that exit, as shown in Fig. 1.

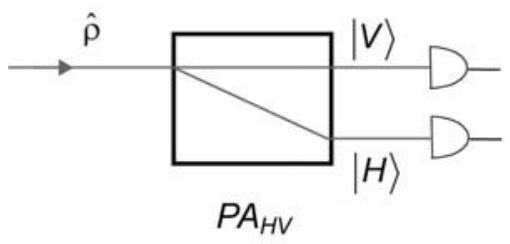

Figure 1. A measurement of polarization in the HV-basis.

If we assign the eigenvalue +1 to horizontal measurements, and -1 to vertical measurements, we can construct a Hermitian polarization operator $\hat{S}_{1}$ in terms of the projection operators onto the $|H\rangle$ and $|V\rangle$ states as

$$
\hat{S}_{1}=(+1)|H\rangle\langle H|+(-1)| V\rangle\langle V|=\left(\begin{array}{cc}
1 & 0 \\
0 & -1
\end{array}\right) .
$$

We can perform polarization measurements in other bases as well. The $\pm 45^{\circ}$ linear polarization states are given by

$$
| \pm 45\rangle=\frac{1}{\sqrt{2}}(|H\rangle \pm|V\rangle) \text {. }
$$

To perform measurements in this basis we replace the $\mathrm{PA}_{\mathrm{HV}}$ in Fig. 1 with a $\mathrm{PA}_{45}$. $\mathrm{A} \mathrm{PA}_{45}$ splits the beam into states polarized at $\pm 45^{\circ}$ with respect to the horizontal axis; this can be accomplished by inserting a half-wave plate whose fast axis is oriented at $22.5^{\circ}$ with respect to the horizontal before a $\mathrm{PA}_{\mathrm{HV}}$. The polarization operator corresponding to the $45^{\circ}$ polarization states is

$$
\hat{S}_{2}=(+1)|+45\rangle\langle+45|+(-1)|-45\rangle\langle-45|=\left(\begin{array}{ll}
0 & 1 \\
1 & 0
\end{array}\right) .
$$

We can also perform measurements in the basis of left- and right-circular polarization states. These states are given by 


$$
|L\rangle=\frac{1}{\sqrt{2}}(|H\rangle+i|V\rangle), \quad|R\rangle=\frac{1}{\sqrt{2}}(|H\rangle-i|V\rangle) .
$$

Such measurements are performed using a circular polarization analyzer $\left(\mathrm{a} P \mathrm{~A}_{\mathrm{C}}\right)$, which is obtained by inserting a quarter-wave plate whose fast axis is oriented at $45^{\circ}$ with respect to the horizontal before a $\mathrm{PA}_{\mathrm{HV}}$. The circularpolarization operator is given by

$$
\hat{S}_{3}=(+1)|L\rangle\langle L|+(-1)| R\rangle\langle R|=\left(\begin{array}{cc}
0 & -i \\
i & 0
\end{array}\right)
$$

Finally, we will find it convenient to express the identity operator as $\hat{S}_{0}$ :

$$
\hat{S}_{0}=\left(\begin{array}{ll}
1 & 0 \\
0 & 1
\end{array}\right) .
$$

The expectation value $\left\langle\hat{S}_{j}\right\rangle$ of a measurement of $S_{j}$ for photons prepared in the state $\hat{\rho}$ is given by

$$
\left\langle\hat{S}_{j}\right\rangle=\operatorname{Tr}\left(\hat{S}_{j} \hat{\rho}\right) .
$$

Note that because the density operator is normalized [Eq. (2)], $\left\langle\hat{S}_{0}\right\rangle=1$. Those familiar with measurement of polarization may recognize the expectation values $\left\langle\hat{S}_{j}\right\rangle$ as the (normalized) Stokes parameters of the beam [12].

It is straightforward to show that the density operator can be written as a linear combination of the operators $\hat{S}_{j}$ :

$$
\hat{\rho}=\frac{1}{2} \sum_{j=0}^{3}\left\langle\hat{S}_{j}\right\rangle \hat{S}_{j} .
$$

Since the $\hat{S}_{j}$ 's are Hermitian operators, and correspond to observables, the expectation values $\left\langle\hat{S}_{j}\right\rangle$ are measureable quantities. Once they have been measured, the density matrix can be determined using Eq. (10).

Thus, the procedure for determining the density matrix of a beam of individual photons is as follows. Start by passing the beam through a polarization analyzer that splits a beam into its horizontal and vertical components $\left(\mathrm{a} \mathrm{PA}_{\mathrm{HV}}\right)$ and then count the photons that exit, as shown in Fig. 1. Assign a value of +1 to photons measured to have horizontal polarization, and a value of -1 to photons measured to have vertical polarization; averaging these measurements yields $\left\langle\hat{S}_{1}\right\rangle$. Now replace the $\mathrm{PA}_{\mathrm{HV}}$ with a $\mathrm{PA}_{45}$ and perform measurements in the $\pm 45^{\circ}$-basis, which enables the determination of $\left\langle\hat{S}_{2}\right\rangle$. Another set of measurements performed using a $\mathrm{PA}_{\mathrm{C}}$ enables the determination of $\left\langle\hat{S}_{3}\right\rangle$. Using $\left\langle\hat{S}_{0}\right\rangle=1$ and Eq. (10) determines the density matrix.

In order to determine the state we must perform measurements corresponding to more than one observable (in this case polarization measurements in three different bases). Furthermore, for each observable we must measure an ensemble of many identically prepared particles in order to accurately determine probabilities. The fact that one must perform many measurements corresponding to different observables is a general property of all quantum state measurement procedures.

\section{QUANTUM STATE MEASUREMENT--EXPERIMENTS}

\subsection{Apparatus}

The experimental apparatus is depicted in Fig. 2. A $405 \mathrm{~nm}, 150 \mathrm{~mW}$ laser diode is used as the pump. The light from this laser first passes through a half-wave plate and then a birefringent plate, before being incident on a pair of Type I BBO 
downconversion crystals, whose axes are oriented at $90^{\circ}$ with respect to each other. This source produces pairs of photons at $810 \mathrm{~nm}$, whose polarization state can be adjusted to be an arbitrary linear combination of $|H H\rangle$ and $|V V\rangle$ :

$$
|\psi\rangle=a|H H\rangle+b e^{i \theta}|V V\rangle \text {. }
$$

The ratio $a / b$ is adjusted by using the half-wave plate to rotate the pump polarization, and the birefringent plate isused to adjust the relative phase between the horizontal and vertical components of the pump, and hence that of the downconverted photons as well [13].

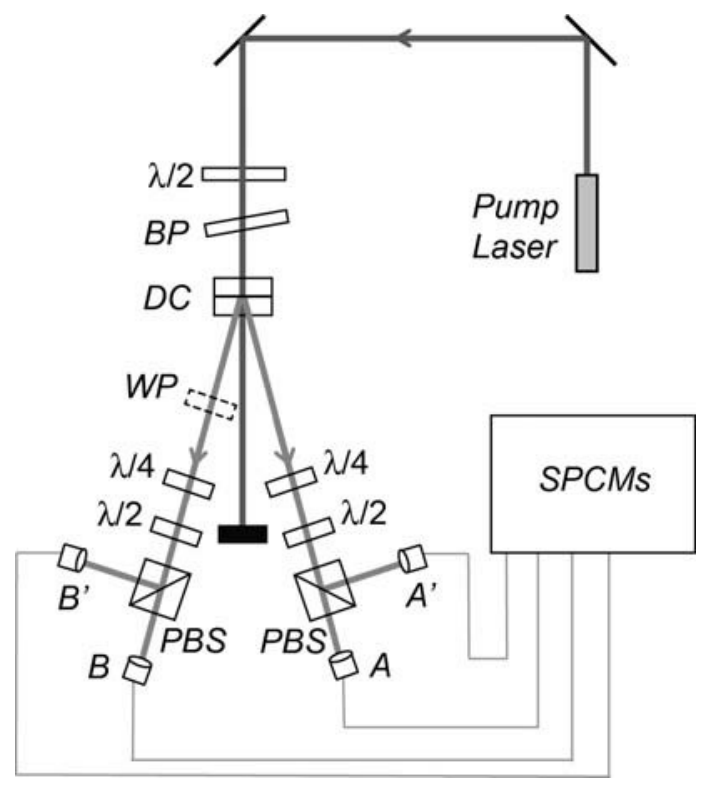

Figure 2. The experimental apparatus. Here $\lambda / 2$ denotes a half-wave plate, $\lambda / 4$ denotes a quarter-wave plate, BP denotes a birefringent plate, DC represents downconversion crystals, WP denotes an optional wave plate, and PBS denotes a polarizing beam splitter. A, A', B, and B' are lenses which focus light into optical fibers, which connect to the single photon counting modules (SPCMs).

The signal and idler beams pass through wave plates, polarizing beam splitters (PBS), and lenses which focus the beams into multimode fibers which deliver the light to single photon counting modules (SPCM). In line with the fibers are RG780 filters which pass the downconversion, but remove scattered pump light. In our experiments detectors A and A' are monitoring the idler beam; measurements on this beam project the signal beam into a single photon state, which is then detected by detectors B and B'. It is the state of this conditionally-prepared signal beam that is measured.

The combination of a quarter-wave plate, a half-wave plate, and a PBS can be used to create a $\mathrm{PA}_{\mathrm{HV}}, \mathrm{a} \mathrm{PA}_{45}$ or a $\mathrm{PA}_{\mathrm{C}}$, depending on the rotation angles of the wave plates. Thus, the wave plates in the idler beam are used to project the beams at detectors A and A' into particular states. For a given setting of the idler-beam wave plates, measurements are then performed with the signal-beam wave plates set to three different combinations, in order to obtain the data required to determine the state of the signal beam.

\subsection{Non-entangled states}

In the first experiments we adjust the source to produce photons in the non-entangled state $|H H\rangle$. In this state the signal-beam photons emerging from the downconversion crystal are in the state $|H\rangle$, independent of any measurement performed on the idler beam. We adjust the wave plates in the idler beam to project the idler photons into state $|+45\rangle$ at A and $|-45\rangle$ at $\mathrm{A}^{\prime}$. We choose these states for idler-beam measurements in order to create equal numbers of photons at $\mathrm{A}$ and $\mathrm{A}^{\prime}$. 
We transform the signal photons from $|H\rangle$ to $|L\rangle$ by inserting a quarter-wave plate into the signal beam (WP in Fig. 2), and then measure the state of the photons in this beam. Real and imaginary parts of the measured density matrix are shown in Fig. 3. This figure shows results for state conditioned on three different measurements performed on the idler beam: detection at $\mathrm{A}$, detection at $\mathrm{A}^{\prime}$, and detection at either $\mathrm{A}$ or $\mathrm{A}^{\prime}$. Note that all three measured states are approximately the same, and all closely resemble the density matrix for the state $|L\rangle$, as expected.

(a)
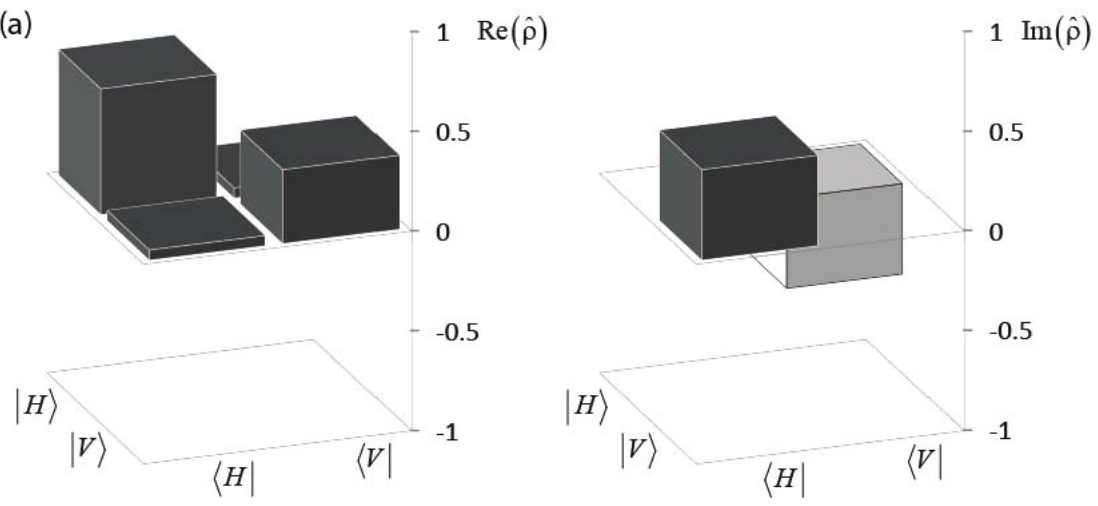

(b)

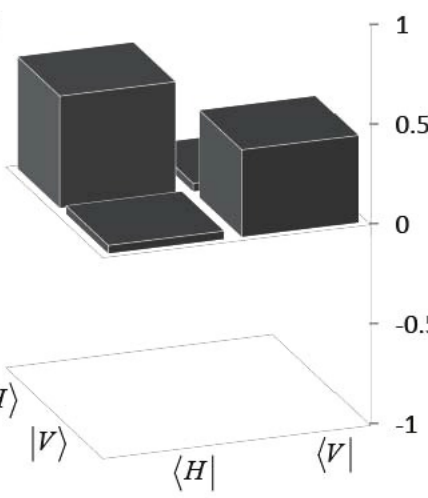

$1 \operatorname{Re}(\hat{\rho})$

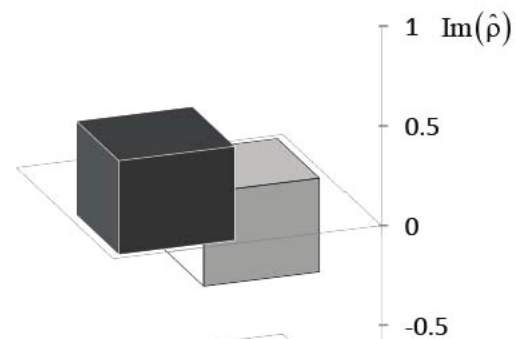

(c)

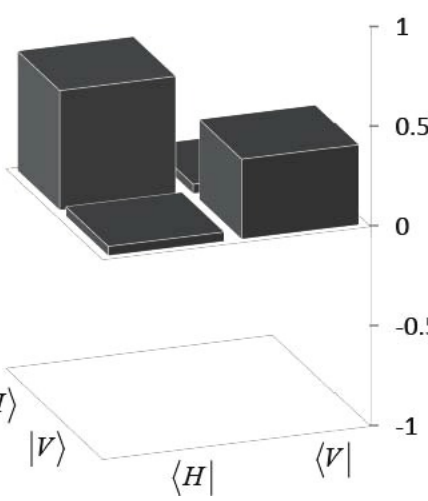

$|H\rangle$

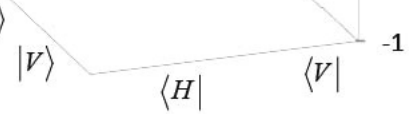

$1 \operatorname{Re}(\hat{\rho})$

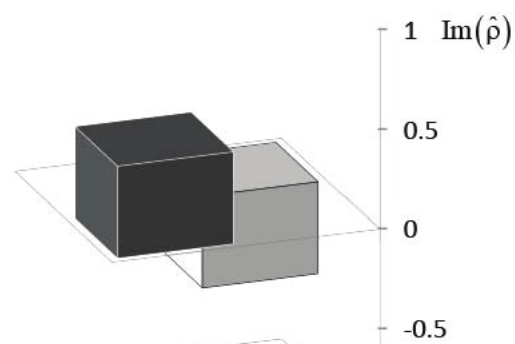

Figure 3. The real and imaginary parts of the density matrix for the signal beam prepared in state $|L\rangle$. The measurements were conditioned on idler detections at detector (a) A, (b) A', (c) A or A'. Darker colored boxes correspond to positive values, while lighter colored boxes correspond to negative values. 
One way to quantify the amount of overlap between two quantum states is to use the fidelity $F$, which is defined as [11]

$$
F\left(\hat{\rho}_{1}, \hat{\rho}_{2}\right)=\left[\operatorname{Tr}\left(\sqrt{\sqrt{\hat{\rho}_{1}} \hat{\rho}_{2} \sqrt{\hat{\rho}_{1}}}\right)\right]^{2}
$$

The fidelity is 1 if the states are identical, and 0 if the states are orthogonal. If the state shown in Fig. 3(a) is compared to $|L\rangle$, the fidelity is $F=0.95$.

\subsection{Entangled states}

We have also performed experiments in which the source produced photons in the entangled Bell state

$$
\left|\phi^{+}\right\rangle=\frac{1}{\sqrt{2}}(|H H\rangle+|V V\rangle) .
$$

The wave plates in the idler beam are adjusted so that the photons at detector A are projected into state $|L\rangle$, and hence the photons at $\mathrm{A}^{\prime}$ are projected into state $|R\rangle$. In Fig. 4 we show the measured density matrices, for measurements conditioned on three different results for the idler photon detection. In Fig. 4(a) the state is conditioned on the detection of a left-circularly polarized photon at A. The measured state approximates the state $|R\rangle(F=0.90)$. This is the expected result, because for photons prepared in state $\left|\phi^{+}\right\rangle$, projecting the idler photon onto the elliptical polarization state $|e\rangle$ collapses the signal photon into the state $\left|e^{*}\right\rangle$. In Fig. $4(\mathrm{~b})$ the state is conditioned on the detection of a rightcircularly polarized photon at $\mathrm{A}^{\prime}$. The measured state approximates the state $|L\rangle(F=0.91)$, which is the expected result. Finally, in Fig. 4(c) the state is conditioned on a detection at either A or A'. This measurement reveals no information about the polarization of the idler beam, so we would expect the signal beam to be projected into a mixed state having $50 \%$ horizontally polarized photons and $50 \%$ vertically polarized photons. The measured state very well approximates this state $(F=0.999)$.

\subsection{Discussion}

The most important feature of the data is the difference between the measurements performed on non-entangled states and those performed on entangled states. For the non-entangled states the measured state of the signal beam is independent of measurements performed on the idler beam (Fig. 3). For the entangled state, if the idler beam is projected into the state $|e\rangle$, the signal beam is measured to be in state $\left|e^{*}\right\rangle$ (Fig. 4).

\section{CONCLUSIONS}

We have designed a series of experiments for an undergraduate teaching laboratory, based on measurements of individual photons and entangled-photon pairs, which explore fundamental aspects of quantum mechanics. We have also integrated these experiments into a curriculum for an upper-level undergraduate quantum mechanics course.

We have recently developed a new laboratory which dramatically demonstrates to students the difference between nonentangled states and entangled states. In this laboratory we perform quantum state measurement of a single photon, the signal photon of a signal-idler pair. For non-entangled states the state of the signal photon is independent of measurements performed on the idler. For an entangled state, the measured state of the signal photon depends strongly on the conditioning of the idler beam measurements. 
(a)
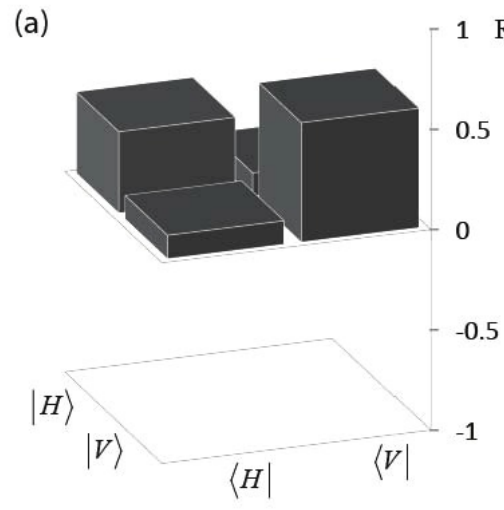

(b)

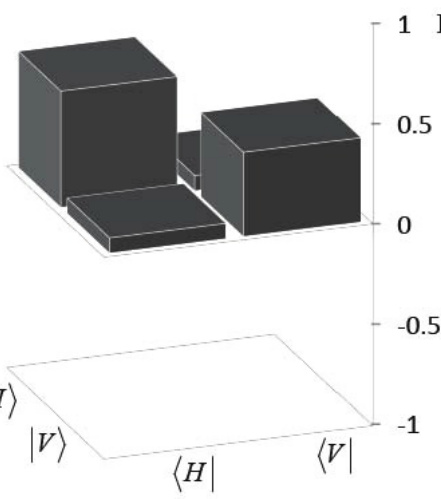

(c)
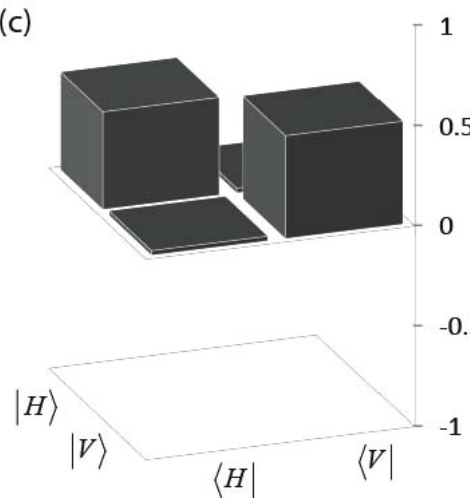
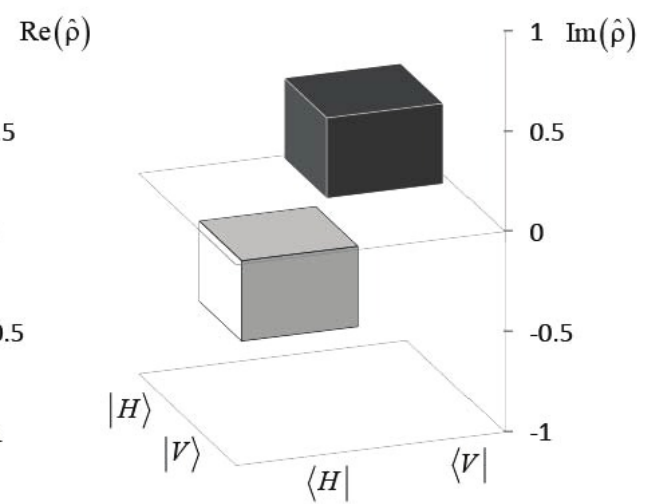

$\operatorname{Re}(\hat{\rho})$
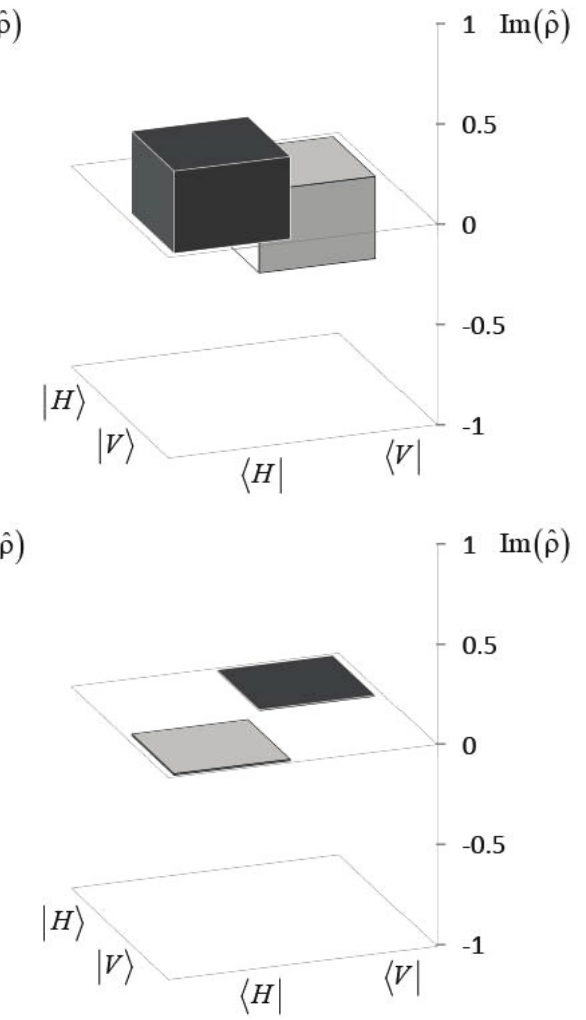

Figure 4. The real and imaginary parts of the density matrix for an entangled-state source. The measurements were conditioned on: (a) A being projected into state $|L\rangle$, (b) A' being projected into state $|R\rangle$, (c) either A or A'. Dark colored boxes correspond to positive values, while light colored boxes correspond to negative values.

\section{REFERENCES}

[1] M. Beck, Quantum Mechanics: Theory and Experiment (Oxford Univ. Press, Oxford, 2012).

[2] J. J. Thorn, M. S. Neal, V. W. Donato, G. S. Bergreen, R. E. Davies, and M. Beck, "Observing the quantum behavior of light in an undergraduate laboratory," Am. J. Phys. 72, 1210-1219 (2004).

[3] E. J. Galvez, C. H. Holbrow, M. J. Pysher, J. W. Martin, N. Courtemanche, L. Heilig, and J. Spencer, "Interference with correlated photons: five quantum mechanics experiments for undergraduates," Am. J. Phys. 73, 127-140 (2005). 
[4] E.J. Galvez and M. Beck, "Quantum Optics Experiments with Single Photons for Undergraduate Laboratories", ETOP 2007 Proc., <http://spie.org/etop/2007/etop07expX.pdf> (2007).

[5] D. Dehlinger and M. W. Mitchell, "Entangled photon apparatus for the undergraduate laboratory," Am. J. Phys. 70, 898-902 (2002).

[6] D. Dehlinger and M. W. Mitchell, "Entangled photons, nonlocality, and Bell inequalities in the undergraduate laboratory," Am. J. Phys. 70, 903-910 (2002).

[7] J. A. Carlson, M. D. Olmstead, and M. Beck, "Quantum mysteries tested: An experiment implementing Hardy's test of local realism," Am. J. Phys. 74, 180-186 (2006).

[8] J. Carvioto-Lagos, G. Armendariz, V. Velazquez, E. Lopez-Moreno, M. Grether, and E. J. Galvez, "The Hong-OuMandel interferometer in the undergraduate laboratory," Eur. J. Phys. 33, 1843-1850 (2012).

[9] D. Branning, S. Bhandari, and M. Beck, "Low-cost coincidence-counting electronics for undergraduate quantum optics," Am. J. Phys. 77, 667-670 (2009).

[10]D. Branning, S. Khanal, Y. H. Shin, B. Clary, and M. Beck, "Scalable multiphoton coincidence-counting electronics," Rev. Sci. Instr. 82, 016102 (2011).

[11] J.B. Altepeter, E.R. Jeffrey, P.G. Kwiat, "Photonic state tomography," in Advances In Atomic, Molecular, and Optical Physics, P.R. Berman and C.C. Lin, eds. (Elsevier, Amsterdam, 2006). p. 105.

[12] E. Hecht, Optics, $4^{\text {th }}$ ed. (Addison Wesley, San Francisco, 2002).

[13] A. G. White, D. F. V. James, P. H. Eberhard, and P. G. Kwiat, "Nonmaximally entangled states: Production, characterization, and utilization," Phys. Rev. Lett. 83, 3103-3107 (1999). 